\title{
Transmission of pandemic influenza H1N1 (2009) in Vietnamese swine in 2009-2010
}

\author{
Karen Trevennec, a,b Lucas Leger, ${ }^{a}$ Faouzi Lyazrhi, ${ }^{\text {b }}$ Eugénie Baudon, ${ }^{c}$ Chung Yan Cheung, ${ }^{d}$ \\ François Roger, ${ }^{a}$ Joseph Malik Sriyal Peiris, ${ }^{c, d}$ Jean-Michel Garcia ${ }^{c}$
}

${ }^{\mathrm{a}}$ French Agricultural Research Center for International Development (CIRAD), Animal and Integrated Risk Management Research Unit (AGIRs), Montpellier, France. ${ }^{b}$ Ecole Nationale Vétérinaire de Toulouse (ENVT), INP, Toulouse, France. ${ }^{\mathrm{C} H K U}$-Pasteur Research Centre, Hong Kong Special Administrative Region, China. ${ }^{\mathrm{d}}$ Department of Microbiology, The University of Hong Kong, Hong Kong Special Administrative Region, China. Correspondence: Karen Trevennec, French Agricultural Research Center for International Development (CIRAD), Animal and Integrated Risk Management Research Unit, Baillarguet Campus, 34398 Montpellier Cedex 5, France. E-mail: carlene.trevennec@cirad.fr

Accepted 9 November 2011. Published Online 30 December 2011.

Background The pandemic of 2009 was caused by an H1N1 (H1N1pdm) virus of swine origin. This pandemic virus has repeatedly infected swine through reverse zoonosis, although the extent of such infection in swine remains unclear.

Objective This study targets small and commercial pig producers in North Vietnam, in order to estimate the extent of H1N1pdm infection in swine and to identify the risk factors of infection.

Methods Virologic and serologic surveillance of swine was carried out in 2009-2010 in pig farms (38 swabs and 1732 sera) and at a pig slaughterhouse (710 swabs and 459 sera) in North Vietnam. The sera were screened using a influenza type A-reactive ELISA assay, and positive sera were tested using hemagglutination inhibition tests for antibody to a panel of H1-subtype viruses representing pandemic (H1N1) 2009 (H1N1pdm), triple reassortant (TRIG), classical swine (CS), and Eurasian avian-like (EA) swine lineages. Farm-level risk factors were identified using a zero-inflated negative binomial model.
Results We found a maximal seroprevalence of H1N1pdm of $55 \cdot 6 \%$ [95\% CI: $38 \cdot 1-72 \cdot 1]$ in the slaughterhouse at the end of December 2009, 2 weeks after the peak of reported human fatalities with H1N1pdm. Farm-level seroprevalence was 29\% [95\% CI: 23·2-35·7]. In seropositive farms, within-herd seroprevalence ranged from 10 to $100 \%$. We identified an increased risk of infection for farms that specialized in fattening and a decreased risk of infection in farms hiring external swine workers.

Conclusions Our findings suggest extensive reverse-zoonotic transmission from humans to pigs with subsequent onward transmission within pig herds.

Keywords Cross-species transmission, epidemiology, influenza pandemic H1N1, swine, Vietnam.

Please cite this paper as: Trevennec et al. (2011) Transmission of pandemic influenza H1N1 (2009) in Vietnamese swine in 2009-2010. Influenza and Other Respiratory Viruses DOI: 10.1111/j.1750-2659.2011.00324.x.

\section{Introduction}

The first human influenza pandemic of 21 st century was an H1N1-subtype virus that emerged through reassortment of North American triple reassortant (TRIG) and Eurasian Avian-like (EA) viruses of swine., ${ }^{1,2}$ The marked antigenic differences between the pandemic and contemporary seasonal $\mathrm{H} 1$ viruses resulted in a large segment of the human population, especially those of younger age, being immunologically naïve to the new pandemic. ${ }^{3}$ The pandemic virus has repeatedly infected swine through reverse zoonosis and has reassorted with other viruses of swine, and this poses a new venue within which novel viruses may emerge to threaten human health. ${ }^{4,5}$ It is therefore important to enhance the surveillance of influenza viruses of swine. As part of a longitudinal study investigating the influenza viruses of swine in North Vietnam, we collected sera from pigs at slaughterhouses and in villages during the winter of 2009-2010. We report here suggestive evidence of extensive transmission of H1N1pdm from human back to pigs.

\section{Material and methods}

\section{Study population}

The Red River Delta (North Vietnam) is characterized by one of the highest animal and human densities within the country with $500 \mathrm{pigs} / \mathrm{km}^{2}$ and 932 person $/ \mathrm{km}^{2}$, respectively, against $83.3 \mathrm{pigs} / \mathrm{km}^{2}$ and 260 person $/ \mathrm{km}^{2}$ at national level (Figure 1A, 1B respectively). ${ }^{6,7}$ Smallholder 


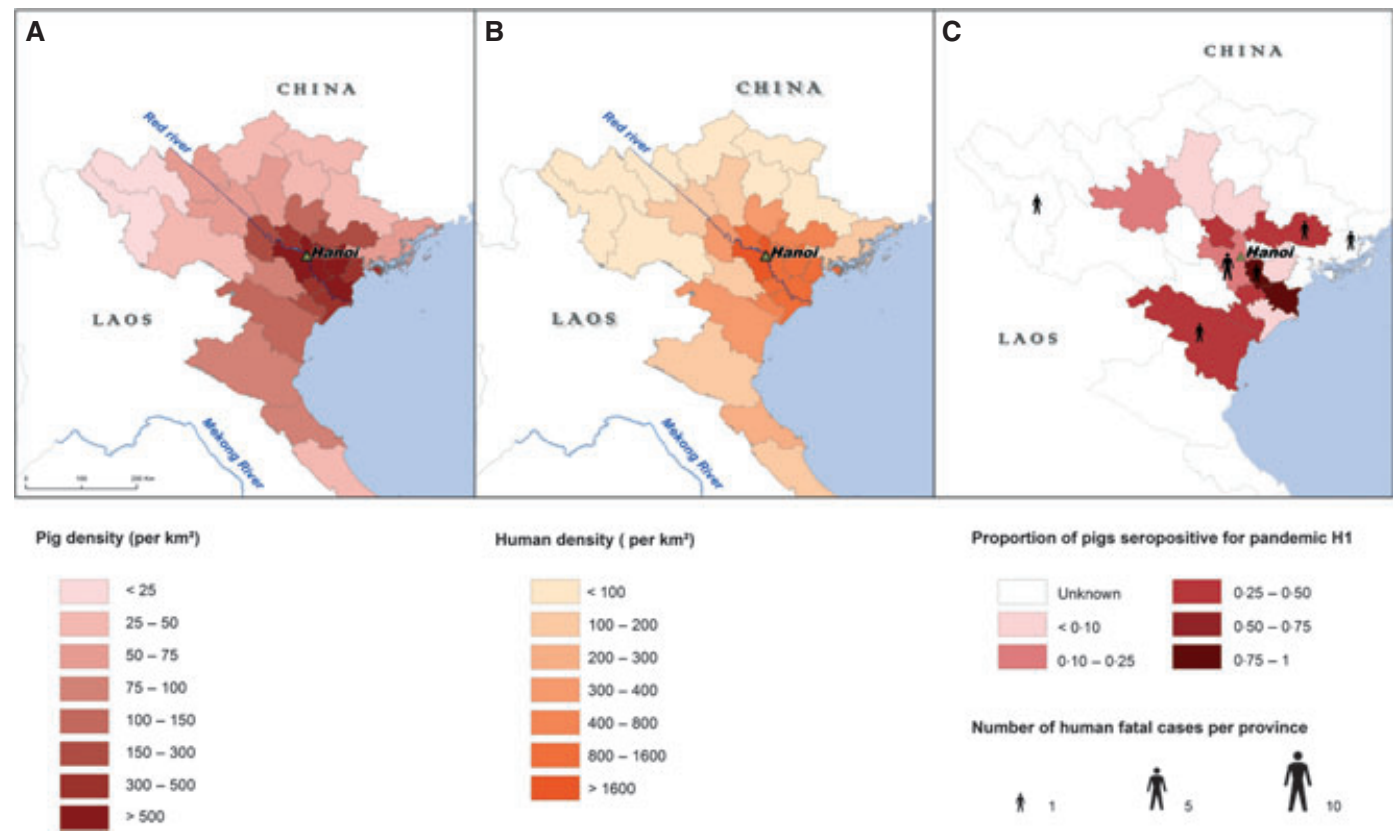

Figure 1. (A) Pig and (B) Human densities in North Vietnam. (C) Seroprevalence of $\mathrm{H} 1$ pandemic in provinces of origin of pigs collected in Hanoi slaughterhouse from October 2009 to May 2010 and human fatal cases during the 2009/2010 epidemic (from September 2009 to February 2010).

systems are dominant and account for approximately $80 \%$ of the national pig population. ${ }^{8}$

\section{Study design and data collection}

\section{Slaughterhouse monitoring}

A survey was performed in Hanoi pig slaughterhouse, which receives animals from the whole Red River Delta. Samples were collected monthly from October 2009 to May 2010, with a doubled frequency of sampling in the winter months December to February. At each visit, 50 pigs were randomly selected by a systematic sampling strategy. The sampling interval was computed on the basis of the number of expected pigs the night of visit, which was provided by the veterinary services. Two tracheal swabs and one blood sample were collected from each animal. A questionnaire was addressed to the pig sellers to record the individual origin and the age of each selected pig.

\section{Survey in pig farms}

Two cross-sectional surveys were performed in pig farms during April 2009 and in the winter 2009-2010. A multistage sampling protocol was applied in six districts, from two provinces: Ha Noi and Bac Giang (only in April 2009). Pig farms were randomly selected from the list of farms provided by local veterinary services or based on a random selection of geographic coordinates. The sample size was calculated using WinEpiscope 2.0 [CLIVE; Royal Dick School of Veterinary Studies, Edinburgh, UK]. The number of farms required to estimate the herd-level seroprevalence of swine influenza was computed on the basis of an expected prevalence $<10 \%$ in April (spring) and 20\% in the winter 2009-2010. ${ }^{9}$ The number of pigs per farm was estimated to detect an expected within-herd seroprevalence of $30 \%$. A total of 122 farms in April and 198 farms in the winter were required. At least 10 pigs per farm had to be collected. When the herd size was smaller than the required sample size, all animals were collected. Animals were randomly selected using a simple sampling strategy when pigs were reared in only one pen and a multistage sampling strategy when age groups were separated. All pigs included in the sample were at least 2 months old in order to avoid any serological reaction because of maternal antibodies. To our knowledge, no vaccination against swine flu was performed in Vietnam. Nasal swabs were collected from animals with respiratory syndromes.

\section{Laboratory assays}

\section{Virologic assays}

Viral isolations from tracheal or nasal swabs were attempted on Madin-Darby canine kidney (MDCK) cells cultured in minimum essential medium (MEM) supplemented with $1 \%$ penicillin-streptomycin (P/S) and $2 \mu \mathrm{g} / \mathrm{ml}$ of 1-tosylamide2-phenylethyl chloromethyl ketone (TPCK)-treated trypsin as described elsewhere. ${ }^{10,11}$ The cells were observed by microscopy for cytopathic effect (CPE) for 7 days. If CPE was detected or otherwise when cells remained CPE negative up to 7 days post-inoculation, the cells were scraped, fixed in $70 \%$ acetone, and stained for influenza A viral antigen using 
DAKO Imagen Flu A antibody (DAKO Diagnostics, IMAGEN Influenza, Dakocytomation, Denmark).

Additionally, all swab samples were tested by RT-PCR as previously described. ${ }^{12}$ Viral RNA were extracted from the swab specimens using the QIAamp Virus BioRobot MDx Kit (Qiagen, GmbH, Hilden, Germany) on the BioRobot Universal System (Qiagen, Invitrogen Life Technologies, Inc., CA, USA) after optimization and validation for use on swab samples. ${ }^{13}$ Random and Uni12 primers were used for cDNA synthesis using Superscript III Reverse Transcriptase (Invitrogen). The BioRobot Universal System was used to set up the reaction mixture, and reverse transcription was performed in a GeneAmp 9700 Thermocycler (Applied Biosystems). Subsequent to the reactions, $20 \mu \mathrm{l}$ of cDNA was diluted $1 / 10$ by adding $180 \mu \mathrm{l}$ of AE buffer (Qiagen) and used for testing on real-time PCR using the LightCycler 480 SYBR Green master mix (Roche) with the primers (forward primer M52C (5'-CTT CTA ACC GAG GTC GAA ACG-3' and reverse primer M253R 5'-AGG GCA TTT TGG ACA AAG/T CGT CTA-3'). The primers was designed to amplify the sequences in the conserved region of influenza $A$ virus matrix gene, thereby detecting viruses from different virus subtypes including swine influenza viruses. ${ }^{12}$ In each assay, serially diluted plasmids containing the full-length $\mathrm{M}$ gene cloned from A/Vietnam/1204/2004 (H5N1) were included as standards to perform absolute quantification. Criteria for samples positive for influenza virus are those with $\mathrm{Cp}$ values $<45$ with a sharp melting curve peak around $85^{\circ} \mathrm{C}$.

\section{Serological assays}

All sera were first screened using ID Screen ${ }^{\circledR}$ (ID-Vet, Montpellier, France) competitive ELISA for influenza A (using nucleoprotein NP as antigen) according to the manufacturer's instructions. The ELISA-positive sera were subsequently tested after receptor-destroying enzyme (RDE) treatment and heat inactivation, using the hemagglutination inhibition (HI) test for H1- and H3-subtype influenza viruses. The $\mathrm{HI}$ tests were performed according to the standard procedures from World Health Organization, using turkey red blood cells. ${ }^{14}$ Viruses representative of different swine virus lineages were selected based on extensive studies in southern China. ${ }^{10,11}$ Four H1-subtype viruses such as swine triple reassortant (A/swine/HK/1110/2006; TRIG), Eurasian avian (A/HK/NS29/2009; EA), classical swine (A/swine/HK/4167/1999; CS), and swine-origin pandemic 2009 virus (A/CA/4/2009; H1N1pdm) and four $\mathrm{H} 3 \mathrm{~N} 2$ viruses such as Eurasian avian-like $\mathrm{A} /$ swine/ HK/1197/02, human A/Sydney/5/97-like swine virus A/swine/HK/2422/1998, contemporary human Brisbanelike $\mathrm{A} / \mathrm{OK} / 483 / 2008$, and $\mathrm{A} /$ swine/HK/2503/2011 were used in this study. They were propagated and titrated following standard procedures on MDCK cells. ${ }^{14}$ Viral titers were calculated by the Reed and Muench method. ${ }^{15}$
If sera had reactions to multiple antigenically related H1-subtype viruses, we categorized a serum as having a homologous reaction profile to $\mathrm{H} 1 \mathrm{~N} 1 \mathrm{pdm}$ positive if that serum had $\geq$ fourfold higher HI titer to H1N1pdm antigen compared with all other H1-subtype viruses. A serum that was seropositive to more than one $\mathrm{H} 1 \mathrm{~N} 1$ virus antigen with titers within fourfold of each other was defined as undetermined $\mathrm{H} 1$ reactivity. A serum that was ELISA-A positive but seronegative to all virus antigens was classified as unknown subtype.

\section{Statistical analyses}

\section{Prevalence estimation}

Animal-level prevalence of virus carriage or seropositivity to H1N1pdm with the associated confidence intervals was computed with the exact binomial method from the EpiTools package using $\mathrm{R}$ version 2.12.0. ${ }^{16-18}$

Seroprevalence of H1N1pdm was computed for each visit at the pig slaughterhouse and mapped in the Red River Delta using Arcview 9.3 ${ }^{\circledR}$ (ESRI, Redlands, CA, USA). The within-herd seroprevalence of H1N1pdm was computed for each farm. Longitude and latitude were used to mark the farm locations and to explore the spatial dependence between seropositive farms. The spatial autocorrelation pattern was described using Moran's I statistic computed at various spatial lags (i.e. the Moran's I statistic was performed for each kilometer from 0 to $10 \mathrm{~km}$ and then for each additional $5 \mathrm{~km}$ from 10 to $20 \mathrm{~km}){ }^{19}$

Results were plotted with the human epidemic curve, which was drawn according to reported cases provided by the Partnership on Avian and Human Pandemic Influenza (PAHI) on the website http://www.avianinfluenza.org.vn/.

\section{Identification of risk factors}

We performed two levels of analyses using $\mathrm{R}$ (version 2.12.0): ${ }^{17}$ one at the province level using the slaughterhouse dataset and one at the farm level using the results of crosssectional surveys performed after the emergence of H1N1pdm in April 2009.

At the province level, the dependant variable was the proportion of seropositive pigs for H1N1pdm. The seroprevalence was tested according to two explicative variables, the pig and human densities. Potential association with both predictors was tested using the Spearman's rank correlation coefficient.

At the farm level, the dependant variable was the count of H1-seropositive pigs within the farm. A total of 14 farm-level potential risk factors of $\mathrm{H} 1 \mathrm{~N} 1 \mathrm{pdm}$ seropositivity and first-degree interactions were investigated. Quantitative variables (number of family members and number of pigs) were categorized when the creation of new biologic or logical variables was possible to correct for the problem of 
linearity. The 14 covariates are presented in Table 1 . The collinearity between categorical variables was tested using the two-sided Fisher's exact test. When two covariates were correlated, they were tested separately in the model selection.

Because there were evidences of overdispersion $\left(\chi^{2}\right.$ test for overdispersion: ${ }^{19} P$ value $>0 \cdot 05$ ), owing to both clustering of animals in herds and an 'excess of zeros', a zeroinflated negative binomial (ZINB) model was computed to assess the associations between the dependant variable and farm-level predictors. ${ }^{18-21}$ The ZINB model performed simultaneously a count model (log link) and a binary model (logit link). The parameter modeled in the count model is the probability of counting $\mathrm{N}$ seropositive pigs within a seropositive farm. The log-transformed number of animals collected in each farm was included in this model, as an additional variable to offset the sample size effect. The parameter modeled in the binary model is the probability of a zero count. ${ }^{18}$ Independent covariates and firstdegree interactions were included in a multivariate ZINB model and selected manually using backward and forward procedures, based on the lowest Akaike's information criterion (AIC). Finally, the Vuong test was performed to check whether the ZINB model fitted the data better than regular negative binomial model. ${ }^{18}$

\section{Results}

\section{Virus isolation in pigs}

Of 710 RT-PCR performed on pig swabs collected in the slaughterhouse and 38 collected in pig farms affected by a respiratory syndrome during the winter 2009-2010, none

Table 1. Farm-level variables as reported by farmers in the winter 2009/2010 in Ha Noi province

\begin{tabular}{|c|c|c|c|}
\hline Variables & Category & Total number of farms & $\begin{array}{l}\text { Percentage } \\
(\%)\end{array}$ \\
\hline \multicolumn{4}{|l|}{ Farm management } \\
\hline \multirow[t]{2}{*}{ Farm type } & Farrowing/fattening & 207 & 29 \\
\hline & Fattening only & & 71 \\
\hline \multirow{2}{*}{$\begin{array}{l}\text { Percentage of familial income provided } \\
\text { by pig production }\end{array}$} & $<50 \%$ & 207 & 81 \\
\hline & $>50 \%$ & & 19 \\
\hline \multirow[t]{3}{*}{ Number of piglets ( $<8$ weeks) } & None & 207 & 69 \\
\hline & $<10$ & & 22 \\
\hline & $>10$ & & 9 \\
\hline \multirow[t]{3}{*}{ Number of growing pigs (8-12 weeks) } & None & 207 & 41 \\
\hline & $<10$ & & 42 \\
\hline & $>10$ & & 18 \\
\hline \multirow[t]{3}{*}{ Number of finishing pigs (>12 weeks) } & None & 207 & 32 \\
\hline & $<10$ & & 29 \\
\hline & $>10$ & & 39 \\
\hline \multirow[t]{3}{*}{ Number of purchase per year } & None & 207 & 54 \\
\hline & $<30$ & & 33 \\
\hline & $>30$ & & 13 \\
\hline \multicolumn{4}{|l|}{ Human-swine interface and sanitary information } \\
\hline \multirow[t]{2}{*}{ Home-made feed (kitchen wastes) } & No & 207 & 33 \\
\hline & Yes & & 67 \\
\hline \multirow{2}{*}{ External employee } & No & 207 & 89 \\
\hline & Yes & & 11 \\
\hline \multirow[t]{3}{*}{ Family members involved in pig production } & One & 207 & 6 \\
\hline & Two & & 69 \\
\hline & More than 2 & & 25 \\
\hline \multirow[t]{2}{*}{ Wear specific clothes and mask } & No & 206 & 46 \\
\hline & Yes & & 54 \\
\hline \multirow[t]{2}{*}{ Disinfect hands } & No & 206 & 100 \\
\hline & Yes & & 0 \\
\hline \multirow[t]{2}{*}{ Visitor restriction } & All allowed & 203 & 91 \\
\hline & Only professionals & & 9 \\
\hline \multirow{2}{*}{$\begin{array}{l}\text { Respiratory syndrome reported in } \\
\text { the last } 12 \text { months }\end{array}$} & No & 207 & 66 \\
\hline & Yes & & 34 \\
\hline \multirow[t]{2}{*}{ Date of sample collection } & December 2009 & 207 & 48 \\
\hline & January 2010 & & 52 \\
\hline
\end{tabular}


were positive. The maximum virus prevalence in slaughterhouse and farms was estimated at $0.52 \%$ and $9 \cdot 2 \%$, respectively (upper limit of confidence intervals).

\section{Seroprevalence to swine influenza viruses}

Samples collected in pig slaughterhouse in Hanoi $(n=459$ pigs $)$ and pig farms $(n=1732$ pigs from 207 farms) gave serological evidence of swine exposure to the H1N1pdm (Figure 2A,B respectively). The prevalence of sera in slaughterhouses having a homologous reaction profile ( $\geq$ fourfold higher titers compared with other $\mathrm{H} 1$ viruses tested) to H1N1pdm overall during the period October 2009 to May 2010 was 97 (21\%) of 459 sera tested. The seroprevalence increased from 6.0\% [95\% CI: $1 \cdot 3-16 \cdot 5]$ in October 2009 to peak at $55 \cdot 6 \%$ [95\% CI: $38 \cdot 1-72 \cdot 1]$ by the end of December 2009 and declined thereafter (Figure 3). Only $11(2 \cdot 4 \%)$ of 459 sera had a homologous titer to any other swine $\mathrm{H} 1$ virus, viz Eurasian avian-like swine (EA), triple reassortant (TRIG), or classical swine (CS). Of these sera, 48 (10.5\%) had reactivity to H1N1pdm and also had comparable (within fourfold) antibody titers to one or more other swine $\mathrm{H} 1$ viruses and are classified as cross-reactive or undetermined H1. The numbers of sera with this serological profile also increased in December 2009, and it is likely that some of these also reflect H1N1pdm infections. The peak of seroprevalence to H1N1pdm in the end of December 2009 followed the peak of human fatal cases by around 2 months.

Pigs collected in Hanoi slaughterhouse came from 13 different provinces, and 11 of these were found to have some H1N1pdm-seropositive animals. As shown on the map (Figure 1C), there was serological evidence that pigs from the whole Red River Delta have been exposed to the H1N1pdm virus.

The estimated farm-level seroprevalence for H1N1pdm was $29 \cdot 5 \%$ [95\% CI: $23 \cdot 3-35 \cdot 7$ ] in the Red River Delta during the winter 2009-2010. The location of exposed farms was scattered in the whole study area (Figure 4). The number of pig sera collected in each farm ranged from 3 to 10 .
Figure 2. Serological distribution of samples from (A) slaughterhouse or (B) farm in the Red River Delta during the winter 2009-2010.

A

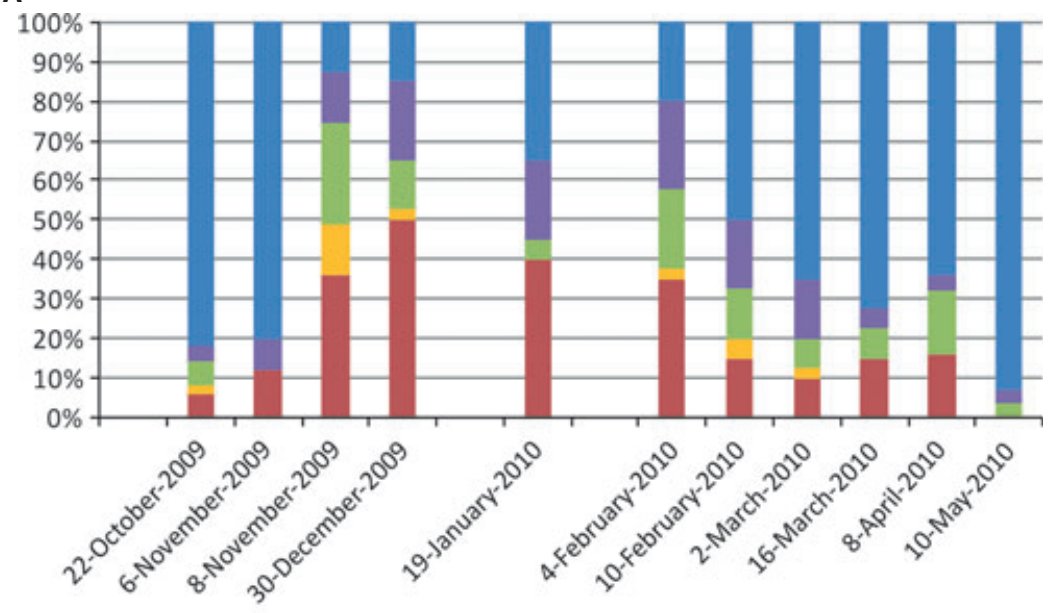

B

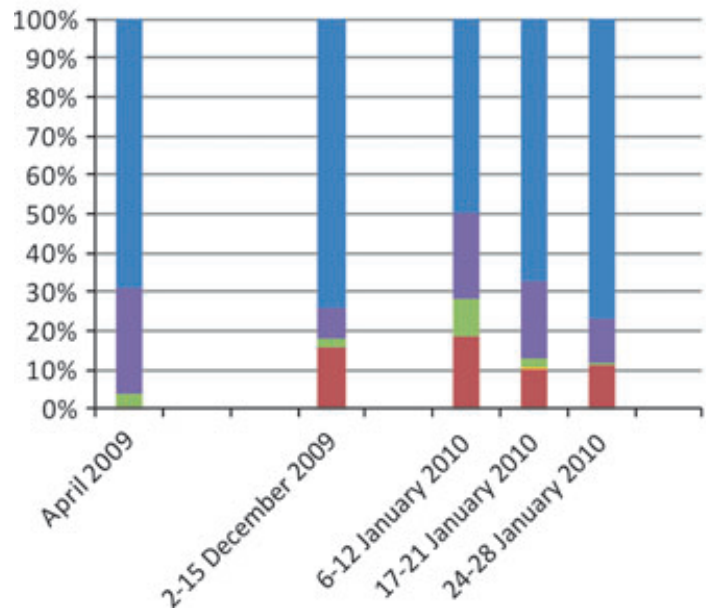

- Elisa negative

Unknown subtype

= Undetermined $\mathrm{H} 1$

ather $\mathrm{H} 1 \mathrm{~N} 1$

- H1N1pdm 


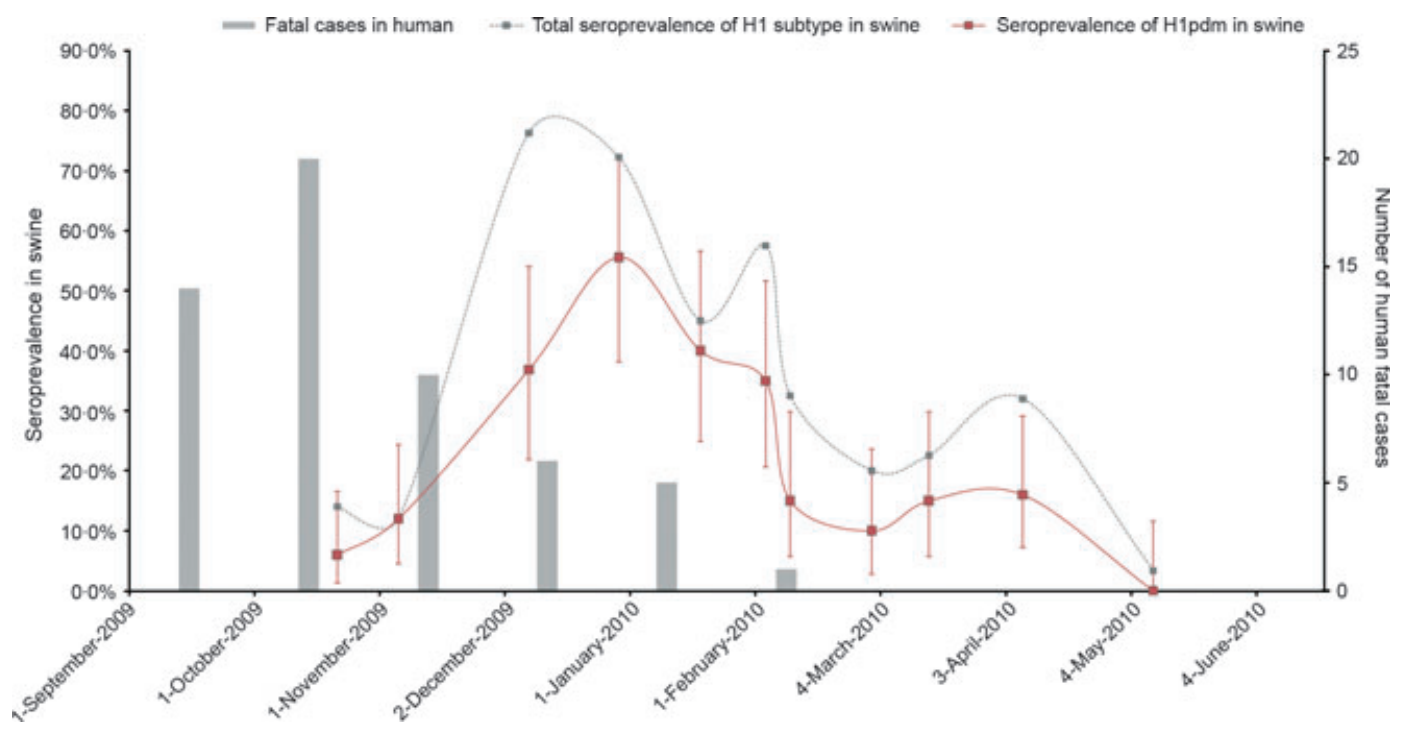

Figure 3. Seroprevalence swine influenza $\mathrm{H} 1$ all subtypes and $\mathrm{H} 1 \mathrm{~N} 1 \mathrm{pdm}$ spreading in pigs collected in Hanoi slaughterhouse from October 2009 to May 2010 and monthly incidence of fatal cases in human in Vietnam.

Among seropositive farms, the within-herd seroprevalence for H1N1pdm was estimated on average at $45 \%$ with a minimum of $10 \%$ and a maximum of $100 \%$. The Moran's I statistic remained closed to zero and insignificant from 0 to $20 \mathrm{~km}$, demonstrating the absence of spatial autocorrelation between seropositive farms.

\section{Risk factors of $\mathrm{H} 1$ pandemic seropositivity}

At the province level, pig and human densities in the Red River Delta were not associated with the seroprevalence of H1N1pdm in pigs. The correlation coefficients were estimated at 0.38 for both variables, and Spearman's correlation tests were not significant $(P$-values $>0 \cdot 20)$. As only one farm stated that they disinfect hands when carrying pigs, this variable was removed from the analysis of risk factors at the farm level.

Fisher's tests showed some correlations between farmlevel variables. Farms specialized in fattening were associated with a large number of finishing pigs $(40.0 \%$ had more than 10 finishing pigs, against $23 \cdot 8 \%$ of farrowing/fattening farms), whereas farrowing/finishing farms had much more piglets $(57 \cdot 1 \%$ had some piglets, against $<2 \%$ of fattening farms). As expected, the purchase was also associated with the type of farm, the number of piglets, and the number of finishing pigs. The purchase of small quantities of pigs ( $<30$ per year) was also correlated with the report of respiratory syndrome diseases. In addition, farms where a large percentage $(>50 \%)$ of familial income was provided by pig production were correlated with the small number of finishing pigs ( $<10$ pigs). Correlated variables were tested separately in the model selection.
The ZINB model was implemented to investigate the role of the 13 remaining potential risk factors. The final model was selected on the basis of the lowest AIC among various combinations of independent covariates and first-degree interactions selected for both the logistic model and the count model. As shown in Table 2A, farms that are specialized in fattening [OR: $0.35(0 \cdot 17 ; 0.70)]$ were associated with a decreased risk of being free from H1N1pdm, meaning an increased risk of farm infection. In the count model (Table 2B), the employment of external swine workers was associated with a low number of seropositive pigs. The predicted counts fitted the data. The Vuong test had a positive value $(3.99 ; P$ value $<0.01)$, indicating that the ZINB model fitted the data better than regular negative binomial model.

\section{Discussion}

H1N1pdm seroprevalence in swine at the Hanoi slaughterhouse rapidly increased during the winter of 2009, to peak at overall seroprevalence of $55 \cdot 6 \%$ [95\% CI: $38 \cdot 1-72 \cdot 1]$ of all animals tested by the end of December 2009 (Figure 3). As in many other parts of Asia, H1N1pdm infection was introduced to Vietnam in June-July 2009. ${ }^{22}$ The detailed epidemic curve of human H1N1pdm infections in North Vietnam is not yet available. However, the reported numbers of fatal H1N1pdm cases peaked in October-November 2009 (Figure 3) and thus appear to have preceded the peak of seroconversion in swine by 1-2 months. This may well reflect the delay between infection and seroconversion of pigs and also the time interval between their infection in the farms and their sale for slaughter. The farm-level 
Figure 4. Location of seropositive farms for H1N1pdm (cross-sectional survey in the Red River Delta, winter 2009-2010).

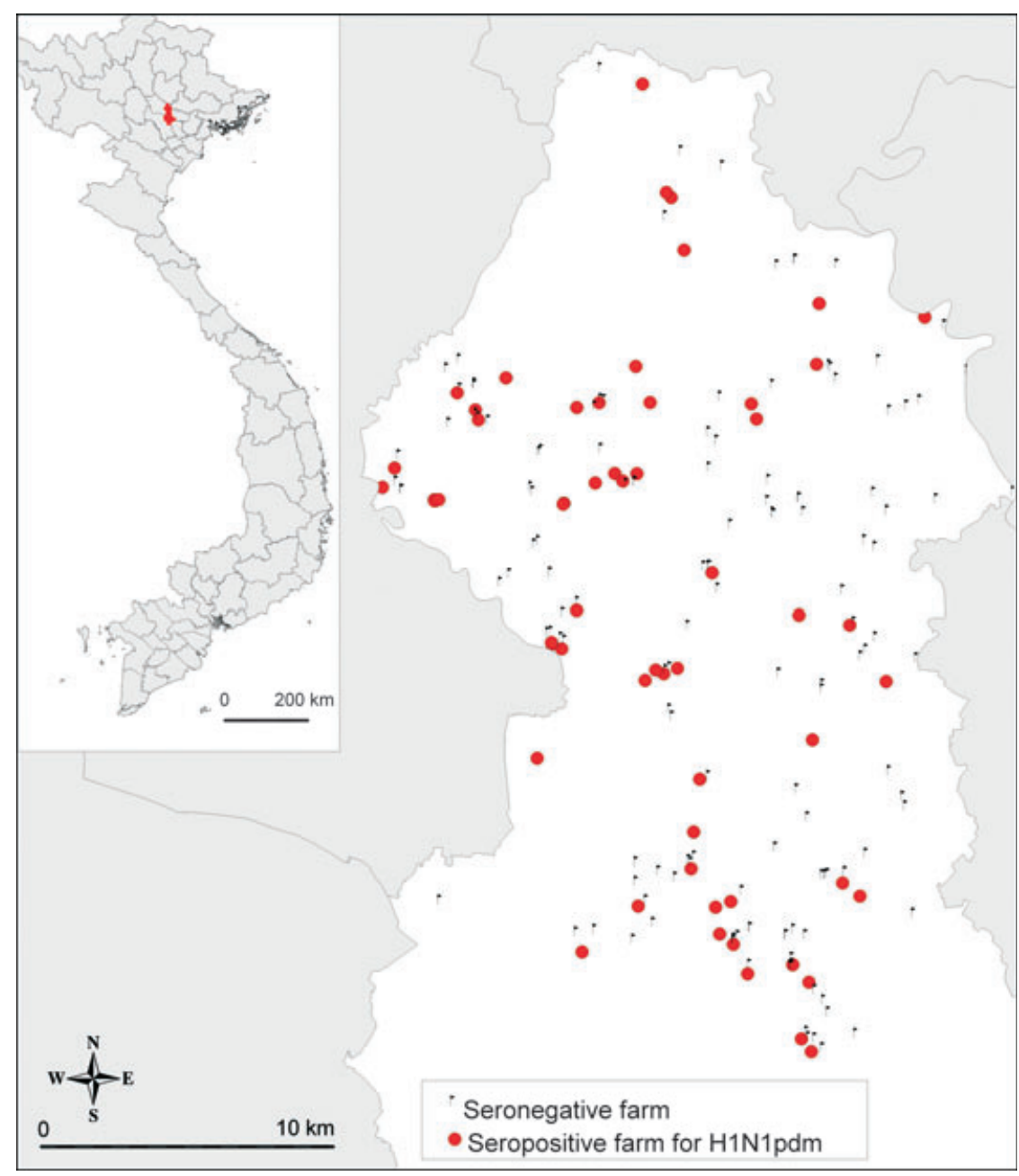

Table 2. Final multivariable ZINB model for H1 pandemic positivity in swine farms of the Red River Delta during the winter 2009-2010. The parameter modeled in the binary model is the probability of a zero count, which represents the probability of a farm being seronegative (A). The parameter modeled in the count model is the probability of counting $N$ seropositive pigs within a seropositive farm (B)

\begin{tabular}{|c|c|c|c|c|}
\hline Variable & Category & OR & $95 \% \mathrm{Cl}$ & $P$ value \\
\hline \multicolumn{5}{|c|}{ (A) Zero-inflated model (logit link) } \\
\hline \multirow[t]{2}{*}{ Type of farm } & Farrowing/fattening & & & \\
\hline & Fattening only & 0.35 & $0 \cdot 17-0 \cdot 70$ & $<0 \cdot 01$ \\
\hline Variable & Category & Estimate & SE & $P$ value \\
\hline \multicolumn{5}{|c|}{ (B) Count model (log link) } \\
\hline External swine worker & Yes & $-1 \cdot 23$ & 0.4 & $<0.01$ \\
\hline
\end{tabular}

seroprevalence for H1N1pdm was 29.5\%, and within-herd seroprevalence in infected farms ranged from 10 to $100 \%$. The data are therefore suggestive of extensive spill-over of H1N1pdm from humans to swine and efficient transmission of the virus within herds. The low seroprevalence of $\mathrm{H} 1$ viruses in swine prior to November 2009 would have facilitated explosive outbreaks of H1N1pdm infection in swine. However, the lack of geographic 
clustering of infected farms is more compatible with multiple discrete transmission events from humans to swine amplifying within each swineherd but not spreading extensively between swineherds. The geographic overlap in the occurrence of human fatal cases and seroprevalence of H1N1pdm in swine (Figure 1C) corroborates this assumption.

Seroprevalence in swine declined after the peak in December 2009-January 2010, suggesting that the H1N1pdm virus was not sustaining high-level virus transmission in swine. This may reflect the reduction of infection in the source (viz humans) but increasing herd immunity in swine may also contribute to this decline in virus activity in pigs. Pig production in Vietnam peaks prior to the Têt festival in February. The post-Têt decline in the susceptible pig population as well as commercial trade after Têt may have also contributed to the decline in seroprevalence. Seasonal factors may also play a role.

Our results are consistent with cases of human-to-swine H1N1pdm transmissions already observed in farms: Canada, ${ }^{23}$ Thailand, ${ }^{24}$ and Korea ${ }^{25}$ (three independent human-to-swine transmissions). Because we had only serological data, we could not determine whether these transmission events were single or several cross-species transmissions. While our data are suggestive of extensive transmission of H1N1pdm within swine herds, it is also suggesting that virus activity is not self-sustaining at high levels in pigs. Reassortants between H1N1pdm and swine viruses have already been isolated in Asia. ${ }^{4}$ If the spread of H1N1pdm in the Vietnamese swine population continues even at low frequency, this human virus may also reassort in Vietnam with swine viruses, as it has been recently observed for $\mathrm{H} 3 \mathrm{~N} 2{ }^{26}$ Further investigation, including continuous monitoring, molecular epidemiology, and modeling, would be necessary to elucidate such questions.

The differences between seroprevalences estimated in slaughterhouse and farms may be related to a number of possible biases including the clustering of animals at farm level, the age of animals, and geographic location. Pigs sent to the slaughterhouse are older than those collected in farms and have more opportunity to have been infected. The swine sampled in farms originate only from the $\mathrm{Ha}$ Noi province, while pigs sampled in the slaughterhouse come from a broader region of the Red River Delta.

There are a number of limitations in our study which is likely to underestimate the prevalence of H1N1pdm infection in swine. Serological testing of swine sera for H1N1pdm by HI tests was only carried out on sera that were positive in screening influenza type A ELISA assay. The sensitivity of such ELISA assays is likely to be less than ideal, and this would be lead to underestimation of the overall H1N1pdm seropositivity in swine. ${ }^{27}$ There is a proportion of sera (up to $22.5 \%$ in February 2010) that had evidence of influenza type A antibody detected in ELISA tests but were negative for the different antigenic variants of H1-subtype swine influenza viruses. This suggests that other subtypes of influenza may be circulating in swine in Vietnam. We included three H3N2 viruses in our panel of virus antigens, viz Eurasian avian-like H3N2; human-like H3N2 swine viruses isolated in Hong Kong in 1998 with A/Sydney/5/97-like hemagglutinin; ${ }^{11}$ and more recent human H3N2 viruses from 2008, with no evidence of virus activity which was surprising. ${ }^{11} \mathrm{H} 3$-subtype viruses have been reported in swine in China ${ }^{9}$ and Thailand. ${ }^{24}$ More recently, H3N2 viruses (e.g., A/swine/Binh Duong/03_08/2010) have been isolated from swine in South Vietnam with $\mathrm{H} 3$ hemagglutinins that are closely related genetically and antigenically to human $\mathrm{H} 3 \mathrm{~N} 2$ viruses A/New York/365/2004 and A/Wyoming/3/2003 $3^{26}$ and to a recently isolated virus from Hong Kong $\mathrm{A} / \mathrm{swi}$ ne/HK/2503/2011 (H3N2). Interestingly, in our study carried out in North Vietnam, none of the pigs have evidence of antibody to A/swine/HK/2503/2011 (H3N2).

Virus isolation attempts from 748 swabs collected during this study did not yield virus isolates. This may in part be related to freezing and thawing of these swabs and also poor-cold chain management as viral isolation could not be carried out at the local laboratory. On the other hand, another recent study of swine influenza in Vietnam found detectable virus only in two pooled swabs of 759 tested, both coming from the same farm. ${ }^{26}$ This and other studies suggest that virus isolation rates from swine are low and larger numbers of swabs need to be tested in order to be successful at isolating viruses. Availability of local virus isolates would have allowed us to use better matched strain for the HI serology testing, probably reducing the proportion of samples that were positive for influenza type A antibodies in the ELISA assay but negative in HI tests.

In farms, the risk of seropositive pigs was associated with the presence of external employees. This is in fact counterintuitive as one would expect that a more heterogeneous work-force will lead to increased risk of introduction of human H1N1pdm infection to swine. Unfortunately, our epidemiological survey data were not precise enough to propose a more detailed explanation for this observation; for example, are employed swine workers more respectful of biosecurity, do they use more self-protection, or are they less inclined to work when they are sick?

Between-farm transmission may occur either via humans (interspecies) or pigs (intraspecies). To our knowledge, no previous study has reported farm-level seroprevalence or risk factors of H1N1pdm in swine. A farm may be infected by infected humans, swine, or fomites. The relatively low proportion of seropositive farms, scattered locations (Figure 4), and the absence of spatial autocorrelation favor limited local diffusion from farm to farm. Thus, the 
observations are in favor of independent farm infections, possibly with infected humans being the major source of infection. However, the number of family members working on the farm, the employment of swine workers, the restriction of visitors, or the wearing of protective clothes/masks was not significantly associated with swine infection risk. The risk factor analyses highlighted an increased risk of farm infection for farms specialized in fattening. Such farms are characterized by the frequent purchase of growing pigs and larger numbers of finishing pigs. Regular introduction of new animals may contribute to the increased infection risk.

\section{Conclusion}

Our seroepidemiological investigations performed in commercial pig farms and a pig slaughterhouse in Vietnam provide evidence that suggests extensive transmission of H1N1pdm from humans to swine and efficient withinherd transmission in infected farms. However, limited evidence of farm-to-farm transmission and the declining seroprevalence in swine by mid-2010 suggests that longterm and sustained maintenance of H1N1pdm in swineherds has so far not occurred. Viral reassortment may of course lead to viruses with greater efficiency in becoming endemic in swine populations. These findings highlight the need for further studies including virus isolation and molecular epidemiology to define the future trajectory of the H1N1pdm virus in pigs and to assess future threats to human health.

\section{Acknowledgements}

This study was partially supported by the GRIPAVI FSP project (MAEE) and Vent d'Est scholarship (MAEE). Laboratory work was also supported by the National Institute of Allergy and Infectious Diseases (NIAID) contract HHSN26600700005C and University Grants Committee of the Hong Kong Special Administrative Region, China (Project No AoE/M-12/06). We would also like to thank Philippe Pourquier from IdVet ${ }^{\circledR}$ laboratory for providing the ELISA kits, Siu K. Ma from The University of Hong Kong for contribution to virologic analyses, and Marie Gély from CIRAD for her help on mapping tools.

\section{References}

1 Garten RJ, Davis CT, Russell CA et al. Antigenic and genetic characteristics of swine-origin 2009 A(H1N1) influenza viruses circulating in humans. Science 2009; 325:197-201.2.

2 Smith GJ, Vijaykrishna D, Bahl J et al. Origins and evolutionary genomics of the 2009 swine-origin H1N1 influenza A epidemic. Nature 2009; 459:1122-1125.
3 Hancock K, Veguilla V, Lu X et al. Cross-reactive antibody responses to the 2009 pandemic H1N1 influenza virus. N Engl J Med 2009; 361:1945-1952.

4 Vijaykrishna D, Poon LL, Zhu HC et al. Reassortment of pandemic H1N1/2009 influenza A virus in swine. Science 2010; 328: 1529.

5 Pereda A, Rimondi A, Cappuccio J et al. Evidence of reassortment of pandemic H1N1 influenza virus in swine in Argentina: are we facing the expansion of potential epicenters of influenza emergence? Influenza Other Respi Viruses 2011; 5:409-412.

6 General Statistics Office Of Vietnam. Population and population density in 2009 by province. GSO: Available at http://www.gso. gov.vn (Accessed 30 June 2011), 2009.

7 General Statistics Office Of Vietnam. Number of pigs by province. GSO: Available at http://www.gso.gov.vn/ (Accessed 30 June 2011), 2008.

8 Kinh LV, Hai LT, Quang DV, Duc NV. Pig production in Vietnam. ACIAR working paper - Centre for international agricultural research - Canberra - Australia, March2002. Priorities for pig research in southeast and the Pacific to 2010. (No. 53): 65-71.

9 Liu W, Wei MT, Tong Y et al. Seroprevalence and genetic characteristics of five subtypes of influenza A viruses in the Chinese pig population: a pooled data analysis. Vet J 2009; 187:200-206.

10 Vijaykrishna D, Smith GJ, Pybus OG et al. Long-term evolution and transmission dynamics of swine influenza A virus. Nature 2011; 473:519-522.

11 Peiris JS, Guan Y, Markwell D, Ghose P, Webster RG, Shortridge KF. Cocirculation of avian H9N2 and contemporary "human" H3N2 influenza A viruses in pigs in southeastern China: potential for genetic reassortment? J Virol 2001; 75:9679-9686.

12 Fouchier RA, Bestebroer TM, Herfst S, Van Der Kemp L, Rimmelzwaan GF, Osterhaus AD. Detection of influenza A viruses from different species by PCR amplification of conserved sequences in the matrix gene. J Clin Microbiol 2000; 38:4096-4101.

13 Ma ESK, Ho PL, Cheung CY et al. Surveillance of influenza viruses in swine in Hong Kong abattoir: methods and feasibility. Influenza Other Respi Viruses 2010; 5(Suppl 1):60-78.

14 Webster R, Cox N, Stohr K. WHO manual on animal influenza diagnosis and surveillance. World Health Organization, Department of Communicable Disease Surveillance and Response. WHO/CDS/ CDR/2002.5 Rev. 1. Available at http://www.who.int/csr/resources/ publications/influenza/en/whocdscsrncs20025rev.pdf (Accessed 25 July 2011).

15 Reed $\mathrm{J}$, Muench $\mathrm{H}$. A simple method of estimating fifty percent endpoints. Am J Hyg 1938; 27:493-497.

16 Aragon T. EpiTools: R Package for Epidemiologic Data and Graphics, 0.5-6 edn. The Comprehensive R Archive Network, Vienna, Austria, 2010.

17 R Development Core Team. R: A Language and Environment for Statistical Computing. Vienna, Austria: R Foundation for Statistical Computing, 2010.

18 Dohoo I, Martin W, Stryhn H. Veterinary Epidemiologic Research. Charlottetown: AVC Inc., 2003.

19 Bivand R, Gentleman R, Gómez-Rubio V, Hornik K, Parmigiani G, Pebesma E, editors. Applied spatial data analysis with R: Springer Science+Business Media, LLC, 2008. 18.

20 Martin TG, Wintle BA, Rhodes JR et al. Zero tolerance ecology: improving ecological inference by modelling the source of zero observations. Ecol Lett 2005; 8:1235-1246.

21 Zeileis A, Kleiber C, Jackman S. Regression models for count data in R. J Stat Softw 2008; 27:1-25. 
22 Hien TT, Boni MF, Bryant JE et al. Early pandemic influenza (2009 H1N1) in Ho Chi Minh City, Vietnam: a clinical virological and epidemiological analysis. Plos Med 2010; 7:e1000277.

23 Forgie SE, Keenliside J, Wilkinson C et al. Swine outbreak of pandemic influenza A virus on a Canadian research farm supports human-to-swine transmission. Clin Infect Dis 2011; 52:10-18.

24 Sreta D, Tantawet S, Na Ayudhya SN et al. Pandemic (H1N1) 2009 virus on commercial swine farm, Thailand. Emerg Infect Dis 2010; 16:1587-1590.

25 Song MS, Lee JH, Pascua PN et al. Evidence of human-to-swine transmission of the pandemic (H1N1) 2009 influenza virus in South Korea. J Clin Microbiol 2010; 48:3204-3211.
26 Ngo LT, Hiromoto Y, Pham VP et al. Isolation of novel triple-reassortant swine H3N2 influenza viruses possessing the hemagglutinin and neuraminidase genes of a seasonal influenza virus in Vietnam in 2010. Influenza Other Respi Viruses 2011; doi: 10.1111/j.17502659.2011.00267.x.

27 Ciacce-Zanella JR, Vincent AL, Prickett JR, Zimmerman SM, Zimmerman JJ. Detection of anti-influenza A nucleoprotein antibodies in pigs using a commercial influenza epitope-blocking enzyme-linked immunosorbent assay developed for avian species. J Vet Diagn Invest 2010; 22:3-9. 